\title{
Hygienemanagement im OP und in der Endoskopie
}

\author{
Nils-Olaf Hübner Marc Thanheiser \\ Fachgebiet 14 "Angewandte Infektions- und Krankenhaushygiene», Robert Koch-Institut, Berlin, Deutschland
}

\section{Schlïsselwörter}

Nosokomiale Infektion - Postoperative Wundinfektion · Infektionsprävention und -kontrolle - Medizinprodukt . Wiederaufbereitung · Endoskopie

\section{Zusammenfassung}

Hintergrund: Postoperative Wundinfektionen gehören zu den häufigsten nosokomialen Infektionen. Die Zunahme von mehrfachresistenten gramnegativen Erregern bereitet gerade in der Viszeralmedizin besondere Sorge. Methode und Ergebnisse: Da nicht alle Risiken für nosokomiale Infektionen beherrschbar sind, können diese Infektionen niemals vollständig vermieden werden. Umso wichtiger ist es, die vorhandenen Maßnahmen konsequent anzuwenden, um den medizinischen Fortschritt, der in der Viszeralmedizin gerade auch durch neue endoskopische und operative Verfahren in den letzten Jahren erkennbar ist, nutzen zu können. Das Verständnis der Erreger und der Infektionsbiologie ist dabei genauso wichtig wie die Kenntnis der nötigen Präventionsmaßnahmen und ihre Einbindung in klinische Prozesse. Bei invasiven Maßnahmen greifen dabei mit dem Patientenweg, dem Weg der Instrumente, der sonstigen OP/Endoskopie-Materialien und Medikamente, der OP-Einrichtung und dem Team mehrere Prozesse ineinander. Schlussfolgerung: Eine optimale Infektionsprävention ist nur bei einer Optimierung aller Prozesse sowie ihrer Interaktion zu erreichen. Anhand einer Übersicht zu Epidemiologie, Risikofaktoren und Möglichkeiten zur Vermeidung von Infektionen in der Viszeralmedizin soll ein Beitrag geleistet werden, um Viszeralmediziner für das Thema Infektionsprävention und Hygiene zu sensibilisieren und die Patientensicherheit zu erhöhen.

\section{Keywords}

Nosocomial infection - Surgical site infection . Infection prevention and control - Medical product . Reprocessing · Endoscopy

\section{Summary}

Infection Prevention in Surgical and Endoscopic

\section{Procedures}

Background: Surgical site infections are one of the most common nosocomial infections. The increasing prevalence of multidrug-resistant gram-negative pathogens as the cause of nosocomial infections is of paricular concern in gastrointestinal medicine and surgery. Method and Results: Nosocomial infections can never be completely avoided, as not all risks of these infections are controllable. All the more, existing infection prevention policies must be consistently applied in order to benefit from the progress that gastrointestinal medicine and surgery have made due to new endoscopic and surgical procedures in recent years. Knowledge of the pathogen and the infection biology are equally important as an understanding of the necessary preventive measures and their integration into clinical processes. Invasive measures are an intersection of multiple processes such as the patient pathway as well as the pathway of surgical instruments, other surgical/endoscopic material and drugs, the operating theatre, and the team. Conclusion: Optimal infection prevention can only be achieved by optimization of all processes and their interaction. Giving an overview on the epidemiology, risk factors, and ways to prevent infections in gastrointestinal medicine and surgery, the authors would like to make physicians and surgeons more aware of infection prevention and hygiene in order to increase patient safety.

\section{KARGER \\ Fax +497614520714}

Information@Karger.com

www.karger.com
(C) 2013 S. Karger GmbH, Freiburg

$1662-6664 / 13 / 0293-0166 \$ 38.00 / 0$

Accessible online at:

www.karger.com/vim
PD Dr. med. habil. Nils-Olaf Hübner

Fachgebiet 14 «Angewandte Infektions- und Krankenhaushygiene» Robert Koch-Institut

Nordufer 20, 13353 Berlin, Deutschland

HuebnerN@rki.de 


\section{Einleitung}

Nosokomiale Infektionen gehören zu den häufigsten Komplikationen bei medizinischen Behandlungen. Etwa jedes fünfte unerwünschte Ereignis bei einer stationären Behandlung ist auf eine nosokomiale Infektion zurückzuführen [1]. Postoperative Wundinfektionen (Surgical Site Infections, SSI) gehören zu den häufigsten nosokomialen Infektionen. Bei einer repräsentativen Punktprävalenzstudie des European Centre for Disease Prevention and Control (ECDC) betrug ihr Anteil an allen nosokomialen Infektionen in Deutschland $24,7 \%$. SSI machten also fast ein Viertel aller nosokomialen Infektionen aus [2].

Zur Prävention von SSI stehen wirksame Instrumente zur Verfügung [3]. Gleiches gilt für die Vermeidung infektiöser Komplikationen im Zusammenhang mit endoskopischen Eingriffen.

Anhand einer Übersicht über die Epidemiologie, Risikofaktoren und Möglichkeiten zur Vermeidung von Infektionen in der Viszeralmedizin soll ein Beitrag geleistet werden, um Viszeralmediziner für das Thema Infektionsprävention und Hygiene zu sensibilisieren und die Patientensicherheit zu erhöhen.

\section{Epidemiologie und Risikofaktoren}

Zur Epidemiologie von SSI stehen Daten aus verschiedenen Quellen zur Verfügung. Neben dem Krankenhaus-Infektions-Surveillance-System (KISS) sind dies unter anderem Daten aus den gesetzlichen Qualitätssicherungsmaßnahmen, aber auch Veröffentlichungen einzelner Kliniken bzw. Zentren und Studien. Die vorhandenen Daten erlauben eine Abschätzung der Gesamtinfektionsraten und der Anzahl von Infektionen auf Basis der Erfassung sogenannter Indikatoroperationen. Im OP-KISS, dem Modul des KISS zur Erfassung von SSI, sind dies mit Bezug auf die Viszeralchirurigie aktuell die Appendektomie, die Cholezystektomie, Eingriffe am Kolon, die operative Therapie der Leistenhernie und Eingriffe am Rektum.

Legt man die Zahlen des KISS mit einer Prävalenz von im Mittel ca. 1,65 Wundinfektionen/100 Operationen für alle erfassten Indikatoroperationen (d.h. nicht nur viszeralchirugische) zugrunde, ergaben sich für Deutschland im Jahr 2008 allein im Zusammenhang mit vollstationären Krankenhausbehandlungen ca. 225000 SSI [4].

Bei genauerer Betrachtung einzelner Eingriffe wird deutlich, dass die SSI-Rate und -Schwere je nach Eingriff und Risikokategorie stark schwankt. Nach den Daten des OP-KISS betrug z.B. die SSI für alle Risikoklassen nach laparoskopischer Appendektomie im Mittel ca. 0,64\%, nach laparoskopischer Cholezystektomie $0,8 \%$ und nach laparoskopischen Eingriffen am Kolon ca. 5\% [5]. Für detaillierte Informationen verweisen wir auf die Internetseite des Nationalen Refe- renzzentrums für Surveillance von nosokomialen Infektionen (www.nrz-hygiene.de).

Für Infektionen nach endoskopischen Eingriffen liegen weit weniger eindeutige Zahlen vor. Hierbei ist zwischen Infektionen, die durch unzureichend aufbereitetes Instrumentarium bedingt sind, und solchen, die durch den Eingriff selbst bedingt sind, zu unterscheiden.

Ein relativ neues Feld sind transendolumenale Eingriffe (Natural Orifice Transluminal Endoscopic Surgery, NOTES). Hierzu sind den Autoren für Deutschland keine systematisch erhobenen Prävalenzdaten von Infektionsraten bekannt.

$\mathrm{Zu}$ bedenken ist, dass insbesondere kleinere operative und endoskopische Eingriffe entweder häufig ambulant durchgeführt werden oder aber die Liegedauer nach dem Eingriff sehr kurz ist. Daher wird eine unbekannte Anzahl infektiöser Komplikationen nicht in der Statistik erfasst.

Neben dem Eingriff an sich haben viele weitere Faktoren entscheidenden Einfluss auf die Infektionsrate, wobei die gegenseitigen Abhängigkeiten und Einflüsse je nach Fall unterschiedlich und nur teilweise verstanden sind. So hat die Technik (offen vs. laparoskopisch) augenscheinlich selbst einen Einfluss auf die Infektionsrate; andererseits ist zu erwarten, dass die Grunderkrankung bzw. die zur Operation führende Erkrankung sowohl die Wahl der Operationsmethode als auch die Infektionsrate beeinflussen. Zur weiteren Stratifizierung von Risikogruppen beim Vergleich von SSIRaten wird der Risikoindex des «National Nosocomial Infections Surveillance (NNIS)»-Systems angewendet [6]. Es wird jeweils ein Punkt für die folgenden Kriterien vergeben: i) die Operation hat länger gedauert als $75 \%$ der Operationen dieser Indikatoroperation; ii) die Wunde ist kontaminiert oder schmutzig; iii) der ASA-Score ist größer als 2. Die Höhe des NNIS-Risikoindex korreliert positiv mit dem Risiko für eine SSI.

Tatsächlich beeinflussen neben operationsbedingten zahlreiche individuelle, patienteneigene Faktoren das Infektionsrisiko. Diese Faktoren lassen sich grob in patienteneigene Risikofaktoren (z.B. hohes Alter (>70 Jahre), Besiedlung mit Staphylococcus aureus oder Methicillin-resistentem S. aureus (MRSA), Immunsuppression, Grunderkrankungen wie Diabetes mellitus, Dialysepflicht, reduzierter Allgemeinzustand oder schlechter Gesundheitszustand, Rauchen, Adipositas, Mangelernährung, Anämie, maligne Erkrankungen, Vorbestrahlung), prozessbedingte Risikofaktoren (z.B. Notfalloperation, Hochrisikooperationen, Dauer des präoperativen Krankenhausaufenthalts), intraoperative Risikofaktoren (z.B. lange Operationsdauer, Handschuhperforation, infizierter Operationsbereich, geringe Erfahrung des chirurgischen Teams, Hypothermie, ausgedehnte Blutungen, Implantation von Fremdmaterial bzw. Einsatz/Ersatz von Implantaten, Nichteinhaltung hygienischer Maßnahmen wie Asepsis oder Antibiotikaprophylaxe) und postoperative Risikofaktoren (z.B. Re-Operation, Drainagen, zentrale Venenkatheter, Blasenkatheter) einteilen [7]. 


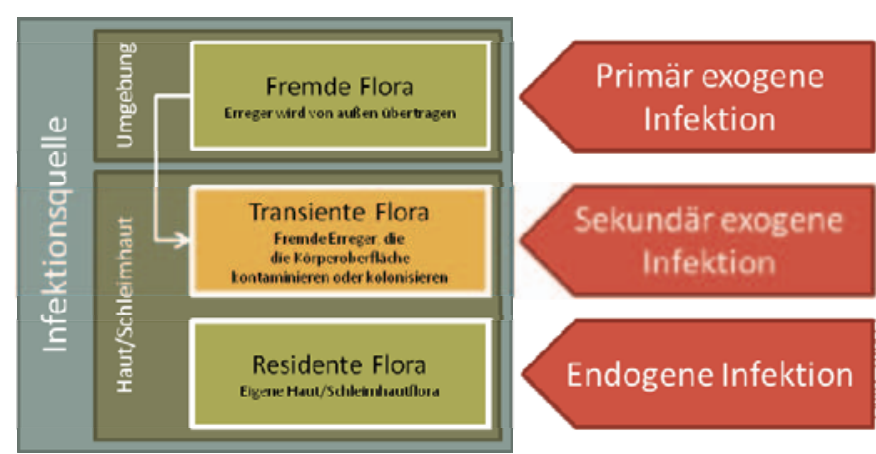

Abb. 1. Bei den exogenen nosokomialen Infektionen stammen die Infektionserreger aus der Umgebung des Patienten. Wichtigste Quellen im Zusammenhang mit Operationen und endoskopischen Eingriffen sind Instrumente/Oberflächen, das OP-Team und die Luft.

\section{Quellen von Erregern postoperativer Wundinfektionen}

Operationen und endoskopische Eingriffe gehören zu den invasivsten medizinischen Maßnahmen. Bei einer großen Zahl endoskopischer Eingriffe wird, wie bei Operationen, die schützende Haut/Schleimhautbarriere durchbrochen. Dabei können Infektionserreger aktiv in das Gewebe eingebracht werden oder durch die Verletzung nachfolgend eindringen. Die Infektionserreger können entweder nach Übertragung von außen direkt zu einer Infektion führen (primär exogene Infektion, z.B. durch kontaminierte Instrumente) oder indirekt zunächst die Haut/Schleimhaut kolonisieren und dann zur Infektion führen (sekundär exogene Infektion, z.B. durch erworbene multiresistente Erreger) oder aus der Haut/ Schleimhautflora des Patienten selbst kommen (endogene Infektionen aus der residenten Flora) (Abb. 1).

Chirurgische Instrumente und sonstige Medizinprodukte stellen eine mögliche Quelle für die iatrogene Übertragung von Krankheitserregern auf den Patienten dar. Bei invasiven Eingriffen können selbst ubiquitär vorkommende, normalerweise apathogene oder fakultativ pathogene Mikroorganismen über damit kontaminierte Medizinprodukte zu Infektionen führen. Aber auch bei nichtinvasiven Eingriffen können Medizinprodukte, die mit pathogenen Mikroorganismen, z.B. vom vorherigen Patienten oder vom medizinischen Personal, kontaminiert sind, den nachfolgenden Patienten schädigen. Die Bandbreite der über Medizinprodukte übertragbaren Krankheitserreger umfasst neben vegetativen Bakterien und Pilzen auch behüllte und unbehüllte Viren sowie bakterielle Sporen und gegebenenfalls Prionen. Das Infektionsrisiko aufgrund von kontaminierten Medizinprodukten ist unter anderem sowohl für chirurgische Instrumente als auch speziell für Endoskope umfangreich beschrieben [8-11].

Das OP-Team kann eine Quelle für Erreger von SSI sein. Die Infektionserreger können dabei entweder aus der Eigenflora des Teams stammen (z.B. MRSA-kolonisiertes Personal) oder Mitarbeiter können als Vektoren Kreuzkontamina- tionen zwischen Patienten hervorrufen. Die Übertragung kann dabei zum einen direkt zur Infektion führen oder sekundär zu einer Quelle für eine spätere Infektion werden. Daher ist nicht nur die Umsetzung von Hygienemaßnahmen während, sondern auch vor und nach dem eigentlichen Eingriff entscheidend. Die Hände des Personals sind der wichtigste Übertragungsweg, insbesondere in Bezug auf multiresistente Erreger. Daher hat die Händedesinfektion eine kaum zu unterschätzende Bedeutung.

Im Vergleich zu den belebten Reservoiren Personal oder Patienten (z.B. Haut, Schleimhäute und Wunden) sowie zu kontaminierten Medizinprodukten und Arzneimitteln ist die Bedeutung der sonstigen Flächen als Quelle von SSI in der Viszeralmedizin nachrangig anzusehen und wissenschaftlich weniger umfangreich untersucht. Es sind jedoch ausbruchartige Erregerübertragungen von Flächen publiziert [12]. Insbesondere Erreger mit hoher Tenazität bzw. mit geringen Infektionsdosen können über kontaminierte Patientenkontaktflächen weiterverbreitet werden [13]. Die Bedeutung der Luft als Quelle von SSI ist weiterhin umstritten. Unstrittig ist, dass Infektionserreger über Aerosole bzw. Tröpfchenkerne übertragen werden können und dass dies zu vermeiden ist. Insbesondere ist das Verspritzen erregerhaltigen Materials zu verhindern. Inwieweit dieser Übertragungsweg bei stringenter Einhaltung der sonstigen Hygienemaßnahmen im OP (z.B. Schutzkleidung einschließlich Mund-Nasen-Schutz, Händeund Flächenhygiene, aseptisches Arbeiten, sterile Medizinprodukte, mikrobiologisch unbedenkliche Raumbelüftung) eine Relevanz bezüglich der SSI gerade in der Viszeralmedizin aufweist, ist ungeklärt.

\section{Wichtige Erreger}

Der wichtigste Erreger von SSI ist nach wie vor S. aureus. Gerade in der Abdominalchirurgie spielen daneben Erreger der Standortflora des Darms eine wichtige Rolle als Infektionserreger. Daher bereitet die Zunahme von mehrfachresistenten gramnegativen (MRGN) Erregern gerade hier besondere Sorge. Candida spp. sind dagegen, wie die in der vorantibiotischen Ära gefürchteten Streptokokken, von untergeordneter Bedeutung [14].

Andere Erreger wie z.B. HIV, Hepatitis A/B/C, Clostridium difficile, Prionen oder Tuberkulose spielen im Zusammenhang mit unzureichend aufbereitetem Instrumentarium eine Rolle $[15,16]$.

In den letzten Jahren hat die Bedeutung implantierbarer, teilimplantierbarer und dauerhaft der Schleimhaut anliegender, häufig endoskopisch platzierter Medizinprodukte wie Prothesen, Grafts, Netzen, Drainagen, Stents usw. stark zugenommen. Diese Medizinprodukte («Devices») bergen neben unbestrittenen neuen Behandlungsmöglichkeiten auch besondere Infektionsgefahren [17]. Diese Produkte bieten eine große und vom Immunsystem nur schlecht zu erfassende 


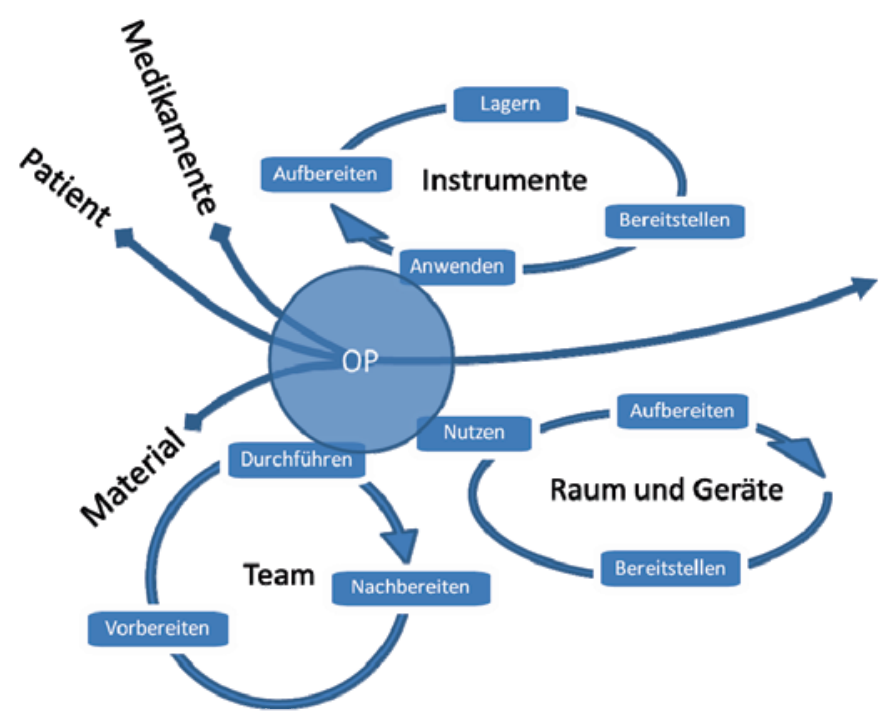

Abb. 2. Bei medizinischen Maßnahmen wie Operationen greifen verschiedene Prozesse ineinander. Nicht jeder dieser Prozesse ist voll beherrschbar, aber in der Optimierung der Einzelschritte und -prozesse sowie der Interaktion der Prozesse liegt ein wesentlicher Ansatz zur Vermeidung postoperativer Infektionen.

Oberfläche, die leicht mikrobiologisch kolonisiert wird. Die sich anschließende Biofilmbildung führt zur Ausbildung von weder vom Körper noch mit Antibiotika zu bekämpfenden Biofilmen, die eine chronische Infektionsquelle darstellen. In diesem Zusammenhang sind Koagulase-negative Staphylokokken (CNS, Stapyhylococcus-epidermidis-Gruppe) als grampositive Bakterien sowie Pseudomonaden und Klebsiellen, aber auch andere gramnegative Bakterien als Infektionserreger von Bedeutung. Dieses Problem betrifft prinzipiell alle Teilbereiche der Implantologie, aber auch der Chirurgie allgemein, da auch Nahtmaterial einen Fremdkörper darstellt, der als Leitstruktur für Infektionen dienen kann [1820]. Auch in der Viszeralmedizin muss daher bei Implantaten, die dauerhaft in sterile Räume eingebracht werden, während des Eingriffs eine mikrobielle Kontamination möglichst ausgeschlossen oder maximal reduziert werden. Bei teilimplantierbaren, vorübergehend applizierten Devices wie Kathetern und Drainagen muss, ähnlich wie bei zentralvenösen Zugängen, vor Anlage und an jedem Liegetag ärztlich entscheiden werden, ob der Nutzen des Device größer ist als das Risiko einer Infektion. Bei Devices mit negativer NutzenRisiko-Bewertung sollte die Indikation kritisch überdacht werden.

\section{Präventionsmaßnahmen}

Zunehmende Kenntnisse einerseits der Übertragungswege und der Eigenschaften der Erreger sowie der Infektionsbiologie und andererseits Kenntnisse über Prozesse, ihre Steuerung und Qualitätssicherung haben zur Entwicklung geeigneter Präventionsmaßnahmen geführt. Die Umsetzung dieser
Tab. 1. Einzelschritte der Aufbereitung von Medizinprodukten

Vorbehandeln
Sammeln
Vorreinigen
Gegebenenfalls Zerlegen
Reinigung
Gegebenenfalls Zwischenspülung
Desinfektion
Spülung
Trocknung
Prüfung auf Sauberkeit/Unversehrtheit
Pflege, Instandsetzung, Funktionsprüfung
Gegebenenfalls Verpackung und Sterilisation
Kennzeichnung
Dokumentierte Freigabe der aufbereiteten Medizinprodukte

Maßnahmen hat einen signifikanten Einfluss auf die Rate nosokomialer Infektionen [3, 21, 22].

Bei der Operation bzw. dem Eingriff greifen dabei mit dem Patientenweg, dem Weg der Instrumente, der sonstigen OPMaterialien/Medikamente, der OP-Einrichtung und dem Team (OP und Anästhesie) mehrere Prozesse ineinander. Eine optimale Infektionsprävention ist nur bei einer Optimierung aller Prozesse und ihrer Interaktion zu erreichen (Abb. 2).

\section{Patient}

Der optimalen Vorbereitung und Nachbetreuung des Patienten kommt große Bedeutung zu, auch wenn das patienteneigene Risiko oft nur eingeschränkt beeinflussbar ist. Wichtige Beispiele für Maßnahmen, die das patienteneigene Risiko senken helfen, sind ein MRSA-Screening und gegebenenfalls Dekolonisierung, Einstellung des Stoffwechsels, Gewichtsreduktion, Einstellung des Rauchens mindestens 30 Tage vor der Operation und der Ausgleich von Mangelernährung [14].

\section{Instrumente}

Die Aufbereitung der Medizinprodukte bewegt sich größtenteils in einem Bereich, der von manchen Juristen als «voll beherrschbares Risiko» angesehen wird und in dem Fehlerfreiheit verlangt wird.

Der Gesetzgeber hat in $\S 4$ Absatz 2 der MedizinprodukteBetreiberverordnung (MPBetreibV) folgendermaßen formuliert: «Die Aufbereitung von bestimmungsgemäß keimarm oder steril zur Anwendung kommenden Medizinprodukten ist unter Berücksichtigung der Angaben des Herstellers mit geeigneten validierten Verfahren so durchzuführen, dass der Erfolg dieser Verfahren nachvollziehbar gewährleistet ist und die Sicherheit und Gesundheit von Patienten, Anwendern oder Dritten nicht gefährdet wird.» Weiterhin wird dort aufgeführt, dass eine ordnungsgemäße Aufbereitung vermu- 
Tab. 2. Mitgeltende Anlagen und weitere Anhänge zur KRINKO-BfArM-Empfehlung
Anlage 1: Zum Begriff «geeignete validierte Verfahren»

Anlage 2: Zu Abschnitt 2.2.3 «Prüfung der technisch-funktionellen Sicherheit»

Anlage 3: Inbetriebnahme und Betrieb von Reinigungs-Desinfektionsgeräten (RDG) zur Aufbereitung von Medizinprodukten (Checkliste)

Anlage 4: Inbetriebnahme und Betrieb von Kleinsterilisatoren zur Aufbereitung von Medizinprodukten (Checkliste)

Anlage 5: Übersicht über Anforderungen an Aufbereitungseinheiten für Medizinprodukte

Anlage 6: Sachkenntnis des Personals

Anlage 7: Maßnahmen zur Minimierung des Risikos einer Übertragung der CJK/vCJK durch Medizinprodukte

Anhang 1 zur Anlage 7: Hinweis auf den Endoskop-Pool an der Universitätsmedizin Göttingen

Anlage 8: Anforderungen an die Hygiene bei der Aufbereitung flexibler Endoskope und endoskopischen Zusatzinstrumentariums

Anhang 6 zur Anlage 8: Zur Aufbereitung flexibler Zystoskope und Bronchoskope

Anhang 7 zur Anlage 8: Aufbereitung von Ultraschallsonden zur Anwendung in der Gynäkologie

Anhang 8 zur Anlage 8: Aufbereitung von Ultraschallsonden mit Schleimhautkontakt tet wird, wenn die gemeinsame Empfehlung der Kommission für Krankenhaushygiene und Infektionsprävention (KRINKO) am Robert Koch-Institut und des Bundesinstituts für Arzneimittel und Medizinprodukte (BfArM) zu den Anforderungen an die Hygiene bei der Aufbereitung von Medizinprodukten beachtet wird [8]. Daher wird diese ursprünglich aus dem Jahr 2001 stammende und im Oktober 2012 aktualisierte Empfehlung den nachfolgenden Ausführungen zugrunde gelegt.

Die Komplexität der Aufbereitung von Medizinprodukten wird deutlich, wenn die Einzelschritte, die nur im Gesamten gesehen eine validierte Aufbereitung darstellen können, aufgelistet werden (Tab. 1).

Die sachgerechte Durchführung dieser Einzelschritte sowie die Rahmenbedingungen für die Aufbereitung werden in der oben genannten Empfehlung beschrieben. Besonders herauszustellende Aspekte sind dabei z.B. die hohe Bedeutung der (nicht fixierenden) Reinigung sowie die Beachtung des notwendigen bakteriziden (einschließlich Mykobakterien), fungiziden, voll viruziden und in bestimmten Einsatzbereichen zusätzlich sporiziden oder prioneninaktivierenden Wirkungsspektrums der eingesetzten Desinfektionsverfahren/-mittel, insbesondere wenn - wie bei semikritischen Medizinprodukten - keine Sterilisation mit feuchter Hitze am Ende der Aufbereitung folgt [23, 24].

Zur Konkretisierung von einzelnen Aspekten der zentralen KRINKO-BfArM-Empfehlung sind dort zusätzlich acht mitgeltende Anlagen und weitere Anhänge enthalten (Tab. 2).

Der Umfang dieser Empfehlung, der insgesamt 67 Seiten beträgt, gilt sowohl im ambulanten als auch im stationären Sektor. Dies macht deutlich, wie wichtig eine fundierte Ausbildung/Sachkenntnis des mit der Aufbereitung beauftragten Personals ist. Einen wichtigen Faktor stellen zudem die Herstellerangaben zur Aufbereitung dar, die - soweit sie plausibel sind - in der jeweiligen Einrichtung umgesetzt werden sollten. Daher sollte bereits vor Anschaffung neuer Medizin- produkte die Aufbereitbarkeit vor Ort mit den für die Aufbereitung zuständigen Mitarbeitern bzw. dem Hygienefachpersonal abgeklärt werden.

\section{Team}

Das Eingriffs- bzw. OP-Team, d.h. das operative und anästhesiologische Team, hat entscheidenden Einfluss auf die Infektionsrate. Infektionspräventive Maßnahmen beginnen dabei schon präoperativ und sind auch in der postoperativen Phase nötig. Wichtige Beispiele sind: perioperative Antibiotikaprophylaxe, Clipping oder Verzicht auf eine Rasur, Verzicht auf Darmreinigung, präoperative Hautantiseptik und erregerdichte OP-Abdeckung, Gewährleistung der Normothermie und gute Narkoseführung. Das Team selbst kann z.B. durch Beachtung der Basishygiene vor, während und nach dem Eingriff, die chirurgische Händedesinfektion und regelmäßigen Handschuhwechsel [25], das Tragen der Bereichs- und sterilen Schutzkleidung und von Haube/ Mund-Nasen-Schutz sowie die Beachtung einer aseptischen, atraumatischen, zügigen Arbeitsweise mit guter Blutstillung, der Entfernung avitalen Gewebes und der strengen Indikationsstellung bei Einbringen von Fremdmaterial hierzu beitragen. Postoperativ sind eine frühe enterale Ernährung und Mobilisierung, Stoffwechselführung, aseptische Technik bei Verbandswechseln sowie die rechtzeitige Entfernung von Drainagen wichtige Elemente zur Vermeidung von Infektionen [14].

\section{Raum und Geräte}

Flächen, z.B. Patientenkontaktflächen, Arbeitsflächen und die Oberflächen medizinischer Geräte, können Vektoren für die Verbreitung unter anderem von multiresistenten Erregern sein. Sie bedürfen daher besonderer Aufmerksamkeit hin- 
sichtlich ihrer Beschaffenheit sowie der Reinigung und gegebenenfalls anschließenden Desinfektion als Teil der Basishygiene.

Die in Hygieneplänen aufzuführenden bereichsspezifischen Maßnahmen sollten zusammen mit dem Hygienefachpersonal ermittelt werden. Als Hilfestellung für die Bewertung kann die örtliche Einteilung nach Risikobereichen mit den jeweils zugehörigen Reinigungs- und Desinfektionsmaßnahmen aus der KRINKO-Empfehlung «Anforderungen an die Hygiene bei der Reinigung und Desinfektion von Flächen» [12] herangezogen werden. Die grundlegenden Anforderungen an Reinigungs- und Desinfektionsverfahren sowie die betrieblich-organisatorischen und baulich-funktionellen Anforderungen sind dort detailliert beschrieben. Ergänzende Informationen finden sich zudem in der Leitlinie «Hygienische Anforderungen an Hausreinigung und Flächendesinfektion» der Arbeitsgemeinschaft der Wissenschaftlichen Medizinischen Fachgesellschaften (AWMF) [26]. Die Deutsche Gesellschaft für Hygiene und Mikrobiologie (DGHM) hat als Fachgesellschaft in den Mikrobiologisch-Infektiologischen Qualitätsstandards (MIQ) Richt- und Grenzwerte für Erregerzahlen auf Oberflächen in medizinischen Einrichtungen formuliert [27].

$\mathrm{Zu}$ den häufig diskutierten Anforderungen an die Raumlufttechnik (RLT) in OP-Räumen hat sich die KRINKO in mehreren Empfehlungen geäußert. Grundsätzlich ist der Einbau einer raumlufttechnischen Anlage in Einrichtungen des Gesundheitswesens nach den anerkannten Regeln der Technik auszuführen. Bezüglich der den örtlichen Gegebenheiten entsprechenden Entscheidung, ob eine (und wenn, welche) RLT-Anlage dort sinnvoll ist, sollte die fachliche Expertise des zuständigen Krankenhaushygienikers genutzt und das zuständige Gesundheitsamt eingeschaltet werden, da diese Einrichtungen der infektionshygienischen Überwachung durch das jeweilige Gesundheitsamt unterliegen und die Handhabung in den Bundesländern teilweise sehr unterschiedlich ist [28]. Dabei ist auch zu bedenken, dass eine RLT verschiedene, d.h. nicht nur infektionspräventive Zwecke erfüllt.

\section{Gesamtkonzepte}

Wie bereits skizziert, können Maßnahmen zur Vermeidung nosokomialer Infektionen nur im Verbund optimal wirken. Infektionsprävention bedeutet daher nicht nur die stringente Anwendung der gebotenen Hygienemaßnahmen im OP, sondern auch die konsequente Nutzung von Möglichkeiten zur Infektionsvermeidung vor und nach dem Eingriff. Entscheidend ist die Erkenntnis, dass medizinische Maßnahmen normalerweise keine isolierten Ereignisse darstellen, sondern mit anderen Maßnahmen im inhaltlichen und zeitlichen Zusammenhang stehen, also einen Teil eines Behandlungspfades bilden. Dies ist die Grundlage der sogenannten «Prozessorientierten Hygiene»: Jede Maßnahme an sich und der gesamte
Pfad werden als Prozess begriffen, der beschrieben, der überwacht und in den gezielt eingegriffen wird [29, 30].

Für die einzelne Maßnahme, z.B. Operation oder Eingriff, bedeutet das die Umsetzung der Bündelstrategie, d.h. die gleichzeitige Einführung verschiedener infektionspräventiver Einzelschritte bei einer medizinischen Maßnahme, um Risiken, die von dieser Maßnahme ausgehen, zu reduzieren [31]. Für den einzelnen Teilprozess, z.B. die Verwendung einer Drainage, greift das Multibarrierenkonzept, das die bei den Einzelmaßnahmen (Anlage, Nutzung und Pflege, Entfernung) nötigen Bündel zeitlich zusammenfügt. Erst die Betrachtung und Kontrolle aller Teilprozesse einer Behandlung, auch über mehrere Behandler und Sektoren hinweg, ermöglichen optimale Sicherheit. Letztlich sind Checklisten ein mächtiges Werkzeug, um auch bei der hohen Komplexität moderner medizinischer Behandlungen Prozessqualität zu sichern [32].

Wie erfolgreich solche, den gesamten Prozess erfassende Hygienekonzepte sind, zeigt die sichere Versorgung mit Lebensmitteln, die ohne das HACCP(Hazard Assessment and Critical Control Points)-Konzept nicht denkbar wäre.

\section{Diskussion}

Vorrangiges Ziel der Krankenhaushygiene ist die Prävention nosokomialer Infektionen. Die Krankenhaushygiene und Infektionsprävention haben seit über 150 Jahren stetig zu einer Senkung von Infektionsrisiken geführt und damit die moderne Medizin erst möglich gemacht. Viele, gerade viszeralmedizinische Maßnahmen jedoch sind durch ihre Invasivität und die Grunderkrankung der Patienten extrem infektionsträchtig. SSI gehören zu den häufigsten, aber auch zu den am besten vermeidbaren nosokomialen Infektionen [1]

Bei den Infektionsrisiken ist klar zwischen denen, die der Patient mitbringt, und denen, die der Eingriff, operativ oder endoskopisch, mit sich bringt, zu unterscheiden. Mit der heute sicher durchführbaren Medizinprodukteaufbereitung kann eine wichtige Ursache nosokomialer Infektionen praktisch umfassend beherrscht werden.

Gerade in den letzten Jahren sind zudem vielversprechende Konzepte entwickelt und erprobt worden, mit denen eine deutliche Senkung der Infektionsrate möglich scheint [3]. Die Innovation liegt dabei weniger in der Entwicklung neuer Maßnahmen, sondern in der Einbettung der Maßnahmen in moderne, prozessorientierte Konzepte [33, 34].

Die prozessorientierte Hygiene als Prozessinnovation kann helfen, die Lücke zwischen evidenzbasierten Empfehlungen und der tatsächlichen Umsetzung zu schließen. Dabei werden die Multibarrieren- sowie die Bündelstrategie aufgegriffen und das erfolgreiche HACCP-Konzept für das Gesundheitswesen umgesetzt. Dies umfasst auch das schnelle Umschalten von der Prävention auf die Behandlung nosokomialer Infektionen und nutzt damit das ganze Verbesserungspotenzial aus [29]. Insbesondere mit der checklistengestützten Bündelung 
und Staffelung von Maßnahmen, d.h. dem prozessorientierten Ansatz der Integration von Hygienemaßnahmen in den Behandlungsweg, steht ein bisher in Deutschland noch nicht ausreichend beachtetes Instrument zur Verfügung, mit dem nicht nur die Compliance mit Hygienemaßnahmen und damit die Wirksamkeit der Infektionsprävention erheblich gesteigert werden kann, sondern auch das Sicherheitsbewusstsein des medizinischen Personals gestärkt wird [32]. Dabei muss der Patient - wie in der Vergangenheit - nicht nur passives «Objekt» sein, sondern kann aktiv eingebunden werden [35].

Durch das novellierte Infektionsschutzgesetz sowie die Verordnungen der Länder besteht eine dichte, moderne und tragfähige rechtliche Grundlage für die sektorübergreifende Umsetzung hygienischer und infektionspräventiver Maßnah- men. Patientensicherheit ist ohne Infektionsprävention nicht denkbar.

Da nicht alle Risiken für nosokomiale Infektionen beherrschbar sind, können diese Infektionen niemals vollständig vermieden werden. Umso wichtiger ist es, die vorhandenen Maßnahmen konsequent anzuwenden, um den medizinischen Fortschritt, der in der Viszeralmedizin in den letzten Jahren gerade auch anhand neuer endoskopischer und operativer Verfahren erkennbar ist, nutzen zu können.

\section{Disclosure Statement}

Für die Autoren bestehen keine Interessenkonflikte.

\section{Literatur}

1 Landrigan CP, Parry GJ, Bones CB, Hackbarth AD, Goldmann DA, Sharek PJ: Temporal trends in rates of patient harm resulting from medical care. N Engl J Med 2010;363:2124-2134.

2 Piening B: Deutsche Daten im Rahmen der ersten europäischen Prävalenzerhebung zum Vorkommen nosokomialer Infektionen und zur Antibiotikaanwendung. Epidemiol Bull 2012;26.

3 Thompson KM, Oldenburg WA, Deschamps C, Rupp WC, Smith CD: Chasing zero: the drive to eliminate surgical site infections. Ann Surg 2011;254:430-436; discussion 436-437.

4 Mielke M, Bölt U, Geffers C: Basisdaten der stationären Krankenhausversorgung in Deutschland unter dem besonderen Aspekt nosokomialer Infektionen; in Bundesamt für Verbraucherschut und Lebensmittelsicherheit, Paul-Ehrlich-Gesellschaft für Chemotherapie, Infektiologie Freiburg (Hrsg): Germap 2010 Antibiotikaresistenz und -verbrauch. Rheinbach, Antiinfectives Intelligence, 2011, pp 141-149.

5 Modul OP-KISS. Referenzdaten. Berechnungszeitraum: Januar 2007 bis Dezember 2011. Berlin, Nationales Referenzzentrum für Surveillance von nosokomialen Infektionen, 2012.

6 Culver DH, Horan TC, Gaynes RP, et al: Surgical wound infection rates by wound class, operative procedure, and patient risk index. National nosocomial infections surveillance system. Am J Med 1991;91:152S-157S.

7 Arbeitskreis Krankenhaus- und Praxishygiene der AWMF: Perioperative Antibiotikaprophylaxe: Leitlinien zur Hygiene in Klinik und Praxis. AWMF online 2012. www.awmf.org/uploads/tx szleitlinien/029-022l_S1_Perioperative_Antibiotikaprophylaxe_2012-02.pdf.

8 Kommission für Krankenhaushygiene und Infektionsprävention, Bundesinstitut für Arzneimittel und Medizinprodukte: Anforderungen an die Hygiene bei der Aufbereitung von Medizinprodukten. Bundesgesundheitsbl Gesundheitsforsch Gesundheitsschutz 2012;55:1244-1310.

$\checkmark 9$ Heudorf U, Exner M: German guidelines for reprocessing endoscopes and endoscopic accessories: guideline compliance in Frankfurt/Main, Germany. J Hosp Infect 2006;64:69-75.
10 Leiß O, Bader L, Mielke M, Exner M: Fünf Jahre Empfehlungen der Kommission für Krankenhaushygiene zur Aufbereitung flexibler Endoskope. Bundesgesundheitsbl Gesundheitsforsch Gesundheitsschutz 2008;51:211-220.

11 Task Force vCJK: Die Variante der CreutzfeldtJakob-Krankheit (vCJK). Epidemiologie, Erkennung, Diagnostik und Prävention unter besonderer Berücksichtigung der Risikominimierung einer iatrogenen Übertragung durch Medizinprodukte, insbesondere chirurgische Instrumente - Abschlussbericht der Task force vCJK zu diesem Thema. Bundesgesundheitsbl Gesundheitsforsch Gesundheitsschutz 2002;45:376-394.

12 Kommission für Krankenhaushygiene und Infektionsprävention: Anforderungen an die Hygiene bei der Reinigung und Desinfektion von Flächen. Bundesgesundheitsbl Gesundheitsforsch Gesundheitsschutz 2004;47:51-61.

13 Kramer A, Schwebke I, Kampf G: How long do nosocomial pathogens persist on inanimate surfaces? A systematic review. BMC Infect Dis 2006;6:130

14 Kommission für Krankenhaushygiene und Infektionsprävention: Empfehlungen zur Prävention postoperativer Infektionen im Operationsgebiet. Bundesgesundheitsbl Gesundheitsforsch Gesundheitsschutz 2007;50:377-393.

15 Archibald LK, Jarvis WR: Health care-associated infection outbreak investigations by the Centers for Disease Control and Prevention, 1946-2005. Am J Epidemiol 2011;174:S47-64.

16 Dancer SJ, Stewart M, Coulombe C, Gregori A, Virdi M: Surgical site infections linked to contaminated surgical instruments. J Hosp Infect 2012;81: 231-238.

17 Lynch AS, Robertson GT: Bacterial and fungal biofilm infections. Annu Rev Med 2008;59:415-428.

18 Alexander J, Kaplan J, Altemeier W: Role of suture materials in the development of wound infection. Ann Surg 1967;165:192-199.

19 Österberg B, Blomstedt B: Effect of suture materials on bacterial survival in infected wounds. An experimental study. Acta Chir Scand 1979;145:431-434.

20 Edmiston CE, Seabrook GR, Goheen MP, Krepel CJ, Johnson CP, Lewis BD, Brown KR, Towne JB: Bacterial adherence to surgical sutures: can antibacterial-coated sutures reduce the risk of microbial contamination? J Am Coll Surg 2006;203:481-489.
21 Liau KH, Aung KT, Chua N, Ho CK, Chan CY, Kow A, Earnest A, Chia SJ: Outcome of a strategy to reduce surgical site infection in a tertiary-care hospital. Surg Infect (Larchmt) 2010;11:151-159.

22 Young B, Ng TM, Teng C, Ang B, Tai HY, Lye DC: Nonconcordance with surgical site infection prevention guidelines and rates of surgical site infections for general surgical, neurological, and orthopedic procedures. Antimicrob Agents Chemother 2011;55:4659-4663.

23 Westermann G, Heeg P: Reinigung - vom Nischendasein zum entscheidenden Prozessschritt. Zentr Steril 2012;20:387-390.

24 Arbeitskreis Viruzidie: Prüfung und Deklaration der Wirksamkeit von Desinfektionsmitteln gegen Viren. Stellungnahme des Arbeitskreises Viruzidie beim Robert Koch-Institut (RKI) sowie des Fachausschusses «Virusdesinfektion» der Deutschen Vereinigung zur Bekämpfung der Viruskrankheiten (DVV) und der Desinfektionsmittelkommission der deutschen Gesellschaft für Hygiene und Mikrobiologie (DGHM). Bundesgesundheitsbl Gesundheitsforsch Gesundheitsschutz 2004;47:62-66.

25 Hubner NO, Goerdt AM, Stanislawski N, Assadian O, Heidecke CD, Kramer A, Partecke LI: Bacterial migration through punctured surgical gloves under real surgical conditions. BMC Infect Dis 2010;10:192.

26 Arbeitskreis Krankenhaus- und Praxishygiene der AWMF: Hygienische Anforderungen an Hausreinigung und Flächendesinfektion. Hyg Med 2010;35: 261-263.

27 Trautmann M, Christiansen B, Häfner H, Lemmen S, Martiny H, Okpara-Hofmann J, Regnath T, Synowzik I, Thomsen J, Wendt C, Zeller E: MIQ 22 Krankenhaushygienische Untersuchungen, Teil I; in Mauch H, Podbielski A, Herrmann M (Hrsg): Qualitätsstandards in der mikrobiologisch-infektiologischen Diagnostik. München, Elsevier, 2005.

28 Kommission für Krankenhaushygiene und Infektionsprävention: Mitteilung der Kommission für Krankenhaushygiene und Infektionsprävention (KRINKO): Kommentar der KRINKO zur DIN 1946-4 (2008). Epidemiol Bull 2010;4 
29 Hubner NO, Flessa S, Haak J, Wilke F, Hubner C, Dahms C, Hoffmann W, Kramer A: Can the hazard assessment and critical control point (HACCP) system be used to design process-based hygiene concepts? GMS Krankenhaushyg Interdiszip 2011;6:Doc24

30 Hubner NO, Flessa S, Jakisch R, Assadian O, Kramer A: Review of indicators for cross-sectoral optimization of nosocomial infection prophylaxis a perspective from structurally- and process-oriented hygiene. GMS Krankenhaushyg Interdiszip 2012; 7:Doc15.

-31 Hübner N: Hygiene muss gelebt werden. Heilberufe spezial 2012;64:10-13.
32 Haynes AB, Weiser TG, Berry WR, Lipsitz SR, Breizat AH, Dellinger EP, Dziekan G, Herbosa T, Kibatala PL, Lapitan MC, Merry AF, Reznick RK, Taylor B, Vats A, Gawande AA; Safe Surgery Saves Lives Study Group: Changes in safety attitude and relationship to decreased postoperative morbidity and mortality following implementation of a checklist-based surgical safety intervention. BMJ Qual Saf 2011;20:102-107.

33 Haynes AB, Weiser TG, Berry WR, Lipsitz SR, Breizat AH, Dellinger EP, Herbosa T, Joseph S, Kibatala PL, Lapitan MC, Merry AF, Moorthy K, Reznick RK, Taylor B, Gawande AA; Safe Surgery Saves Lives Study Group: A surgical safety checklist to reduce morbidity and mortality in a global population. New Engl J Med 2009;360:491499.
4 Weiser TG, Haynes AB, Dziekan G, Berry WR, Lipsitz SR, Gawande AA; Safe Surgery Saves Lives Investigators and Study Group: Effect of a 19-item surgical safety checklist during urgent operations in a global patient population. Ann Surg 2010;251:976-980.

35 Kramer A, Schilling M, Heidecke CD: Infektionspräventions-Check-in und InfektionspräventionsCheck-out zur Prävention nosokomialer Infektionen. Zentralbl Chir 2010;135:44-48. 